\title{
Digital therapeutics and clinical pharmacology
}

\author{
Jae-Yong Chung* \\ 13620, Korea \\ ${ }^{\star}$ Correspondence: J. Y. Chung; Tel: +82-31-787-3955, Fax: +82-31-787-4091, E-mail: jychung@snubh.org
}

Department of Clinical Pharmacology and Therapeutics, Seoul National University College of Medicine and Bundang Hospital, Seongnam

Check for updates

Received 2 Mar 2019

Revised 11 Mar 2019

Accepted 11 Mar 2019

\section{Keywords}

Clinical pharmacology,

Digital therapeutics,

Healthcare

pISSN: 2289-0882

eISSN: 2383-5427
Digital therapeutics (DTx) is a new subsection of digital health that is primarily driven by software and will be of great interest to clinical pharmacologists. In this article, an overview of DTx, including definition, position in the landscape of therapeutics, product categories, benefits, and challenges, is provided. Discussions from the point of view of clinical pharmacology are presented, as DTx should have exposure-response relationships. The principles of clinical pharmacology can be applied to DTx as they are comparable to pharmacotherapy. Clinical pharmacology has great potential in the development, application, and regulation of DTx.

\section{Introduction}

The advances in digital technology have converged with medical science, which has allowed digital health to become a critical part of routine clinical care. Consequently, a new category of therapeutics integrating digital technology called Digital Therapeutics (DTx) has been launched. After the first prescription digital therapeutic for the treatment of substance use disorder (SUD) was approved by the FDA through a de novo submission for classification as a Class II medical device in 2017,[1] the potential use of DTx clinically has since gained remarkable attention. New start-ups are actively working on the development of DTx. The Digital Therapeutics Alliance was founded as a nonprofit trade association made up of industry leaders and stakeholders engaged in evidence-driven advancement of DTx.[2] Because it is uniquely a software-based therapeutic intervention, this new category of therapeutics will be challenging and of great interest to clinical pharmacologists who are accustomed to chemical or biological medicines whose physiological mechanisms involve a specific target. In this article, the concept, development, and regulation of DTx will be discussed, as well as the clinical pharmacologist point of view.

Copyright (C) 2019 Jae-Yong Chung

(a) It is identical to the Creative Commons Attribution Non-Commercial License (http://creativecommons.org/licenses/by-nc/3.0/).

@ (T) This paper meets the requirement of KS X ISO 9706, ISO 9706-1994 and ANSI/NISO Z.39.48-1992 (Permanence of Paper).

Reviewer

This article was invited and reviewed the by the editorial board members of TCP.

\section{Definition of digital therapeutics}

DTx, a subset of digital health, is a health discipline and treatment option that utilizes a digital and often online health technologies to treat a medical or psychological condition.[3] Digital health describes all technologies that engage with patients for health-related purposes. It encompasses a wide range of products used across the wellness and healthcare industry. DTx can be distinguished from other digital health categories based on the primary function of delivering software-generated therapeutic interventions directly to patients to prevent, manage, or treat medical disorders or diseases. DTx and several adjacent areas connected in the digital health landscape are featured in Figure 1.[4]

DTx is often confused with digital medicine, which can be defined as a category of pharmaceuticals that combine a prescription medication with an ingestible sensor component. Fol-

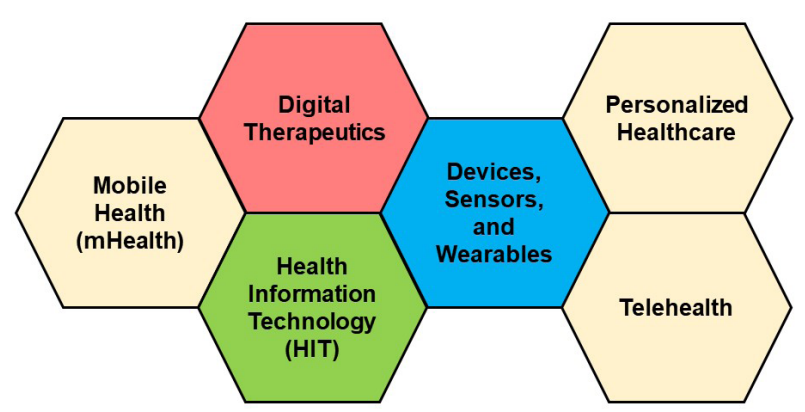

Figure 1. Landscape of Digital Health (Adopted from the Digital Therapeutics Alliance white paper).[4] 
lowing the landmark regulatory approval of digital aripiprazole (Abilify Mycite ${ }^{\mathrm{TM}}$ ) late in 2017, digital medicines have begun the transition from clinical development to mainstream utilization.[5] They are designed to communicate patients' medication administration compliance to mobile and/or web-based applications. Therefore, it is widely applicable to improving patient's adherence when the patient's outcome is important, in conditions such as neuropsychiatric diseases and HIV infection. Proteus Digital Health ${ }^{\odot}$ Inc. had developed Abilify Mycete ${ }^{\mathrm{TM}}$ and is now developing new digital medicines using similar technology, for other therapeutic areas including mental health, cardiovascular and metabolic conditions, infectious diseases, and oncology.[6]

DTx empowers patients, healthcare providers, and payers with intelligent and accessible tools for addressing a wide range of conditions through data-driven interventions. [4] Similar to other medicinal products, the effectiveness of DTx-based products should be scientifically proven before delivery to patients for the prevention, management, or treatment of diseases. DTx can be used independently or in combination with other treatment modalities. Best practices for DTx are related to design, clinical validation, usability, and data security. To support product claims regarding risk, efficacy, and intended use, they are reviewed by regulatory bodies.

DTx is a novel category of therapeutic options. In addition to existing pharmacological, non-pharmacological (surgical, radiation, and physical), and psycho-behavioral therapy, it can cover therapeutic areas that have previously not been well described. However, the current stages of most DTx-based products correspond to the convenient replacement of already existing treatment settings, such as that for cognitive behavioral therapy

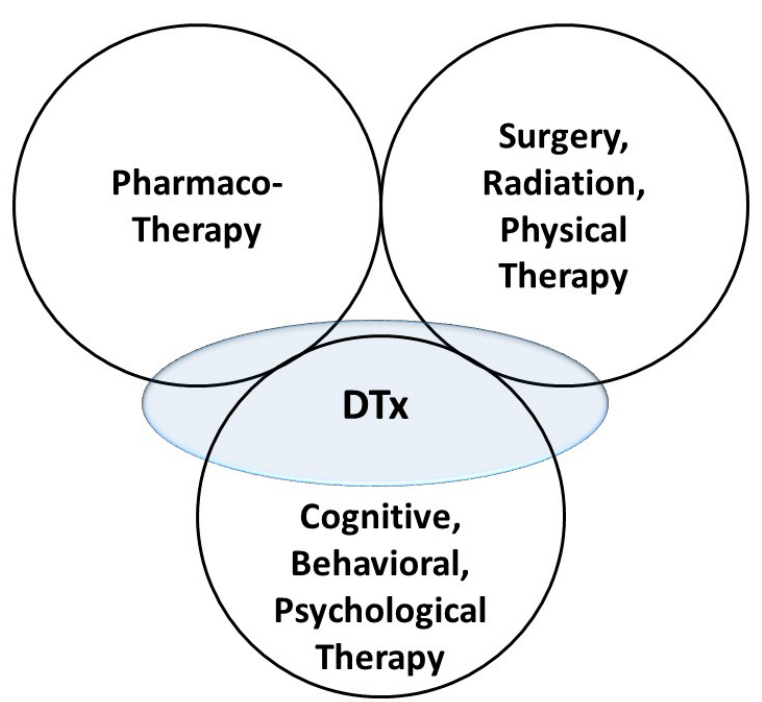

Figure 2. Conceptual position of Digital Therapeutics (DTx) in relation to the existing therapeutic options.
(CBT) and reminiscence therapy. The conceptual position of DTx in relation to the existing therapeutic options is depicted in Figure 2.

In addition to enhancing and supporting current medical treatments, DTx has the potential to provide patients, providers, and payers with novel therapeutic options for unmet medical needs. DTx products can be delivered in a secure manner and can be personalized for each patient. DTx products are more convenient than existing therapies because they can be applied independently of a patient's schedule and location. DTx treatments are more expandable as they provide ubiquitous access to treatment options for diseases or conditions that were previously untreated or undertreated by traditional therapies. For example, DTx would overcome limitations to delivering therapies to patients having stigma associated diseases such as mental disorder, AIDS, venereal diseases, leprosy, and certain skin diseases. Secure data provided by DTx are integrable into provider portals and clinical data warehouses so that they can be utilized for patients' personalized therapy and/or for clinical research and development.

\section{Categories of digital therapeutics and product examples}

The Digital Therapeutics Alliance adopted a four-category classification of DTx.[4] Based on its target product profile, each DTx treatment belongs to one of the following categories:

1. Address a medical condition.

2. Manage or prevent a medical disorder or disease.

3. Optimize medication.

4. Treat a medical disease or disorder.

The requirements for using each category of DTx product vary in accordance with the purpose of the product (to support product claim, clinical evidence generation, patient access to product, and relationship to concurrent therapies). For example, when a category $1 \mathrm{DTx}$ product addressing a medical condition requires enabling patient access, it can be done via the directto-consumer pathway, whereas prescriptions are required for a category $4 \mathrm{DTx}$ to be delivered to patients.

There is an increasing number of start-ups launching DTx, and most of their clinical development programs are carried out in the USA. By the end of 2018, the FDA had approved 2 prescriptions requiring DTx (Category 4 ) for substance use disorder. Examples of DTx medications under development or on the market are listed along with their therapeutic area and product category in Table 1. In their current stages, most of them are category 2 or 3 products. All category 4 DTx products on the list are for psychiatric diseases, mainly CBT.

\section{Regulatory process}

The world's first prescription DTx was developed and approved in the US. DTx is regulated by the Center for Devices and Radiological Health (CDRH) at the FDA. The FDA considers DTx submission as mobile medical applications (MMA). 
Table 1. Examples of Digital Therapeutics under development or on the market

\begin{tabular}{|c|c|c|c|c|}
\hline Company name & Product name & Description & Therapeutic area & Category \\
\hline Pear Therapeutics & $\begin{array}{l}\operatorname{reSET}^{\circledR} \\
\operatorname{reSET}^{\circledR}-\mathrm{O}^{\mathrm{TM}}\end{array}$ & $\begin{array}{l}\text { An adjunct therapy to standard, outpatient treatment for } \\
\text { Substance Use Disorder (SUD) }\end{array}$ & CNS & 4 \\
\hline $\begin{array}{l}\text { Propeller Health } \\
\text { \& Boehringer Ingelheim }\end{array}$ & RESPIMAT $^{\circledR}$ & $\begin{array}{l}\text { Combined software and hardware program to improve asthma } \\
\text { and COPD control and optimize healthcare utilization }\end{array}$ & Respiratory & 3 \\
\hline Akili Interactive & AKL-T01 & $\begin{array}{l}\text { Utilizing adaptive sensory stimulus software for the treatment of } \\
\text { ADHD delivered through an engaging video game experience }\end{array}$ & CNS & 4 \\
\hline Dthera Sciences & DTHR-ALZ & $\begin{array}{l}\text { Delivering reminiscence therapy to Alzheimer's sufferers in a } \\
\text { scalable and personalized manner. }\end{array}$ & CNS & 4 \\
\hline Big Health & Spleepio $^{\text {TM }}$ & $\begin{array}{l}\text { Sleep improvement program featuring Cognitive Behavioral } \\
\text { Therapy }(\mathrm{CBT}) \text { techniques. }\end{array}$ & CNS & 2 \\
\hline Cognoa & various & $\begin{array}{l}\text { Al-based digital diagnostics and personalized therapeutics for } \\
\text { pediatric behavioral healthcare. }\end{array}$ & $\begin{array}{l}\text { CNS, } \\
\text { Pediatrics }\end{array}$ & 2 \\
\hline KAIA & Motion coach & $\begin{array}{l}\text { Delivery of physical exercises, behavioral therapy, and education } \\
\text { for chronic back pain patients. }\end{array}$ & $\begin{array}{l}\text { Musculo } \\
\text {-skeletal }\end{array}$ & 2 \\
\hline Glooko & MIDS & Insulin mobile dose calculator for adults with Type 2 diabetes. & Metabolism & 3 \\
\hline WellDoc & BlueStar $^{\circledR}$ & $\begin{array}{l}\text { Engaging individuals with Type } 2 \text { diabetes, hypertension, and } \\
\text { obesity, and their providers, to improve self-management and } \\
\text { outcomes. }\end{array}$ & Metabolism & 2 \\
\hline $\begin{array}{l}\text { Omada Health, Noom, } \\
\text { Etc }\end{array}$ & & $\begin{array}{l}\text { Personalized digital program to help people prevent the onset of } \\
\text { diabetes and other chronic diseases. }\end{array}$ & Metabolism & 2 \\
\hline MedRhythms & & $\begin{array}{l}\text { Neurologic music therapy to address motor, speech, and } \\
\text { cognitive dysfunction caused by neurologic disease or injury. }\end{array}$ & CNS & 2 \\
\hline
\end{tabular}

Category: 1 = Address a medical condition, $2=$ Manage or prevent a medical disorder or disease, $3=$ Optimize medication, and $4=$ Treat a medical disease or disorder. $\mathrm{CNS}=$ Central Nervous System.

There is a specific 'enforcement discretion' policy that does not oblige manufacturers to submit premarket review applications or to register and list their apps with the FDA. Apps that provide easy access to or help patients organize their health information, and help patients self-manage their disease or condition (e.g., coaching and wellness) without providing specific treatment suggestions are designated to this category, irrespective of their therapeutic benefit.[7] MMAs that do not fall under this enforcement discretion policy are classified and regulated as Class I, Class II, or Class III devices, depending on an analysis of safety/risk, intended use, and indications for use. Class III is designated for devices possessing the most significant risk of illness or injury, and these devices are subject to the premarket approval (PMA) review process. For Class II and I devices categorized as special and general controls respectively, a $510(\mathrm{k})$ premarket submission is required. A 510(k) compares the device to one or more similar devices that are already legally marketed and claims the new device to be substantially equivalent. If no such marketed devices exist, a de novo submission for a Class I or II device must be made. For example, Pear Therapeutics' reSET ${ }^{\oplus}$ for the treatment of substance use disorder was approved through a de novo submission for a Class II medical device.[1] Also, their reSET-O ${ }^{\mathrm{TM}}$ went through the expedited access pathway and was reviewed under a $510(\mathrm{k})$, claiming substantial equivalence to the already approved reSET ${ }^{\circ}$.[8]

\section{Challenges}

The challenges of DTx are mainly related to the generation of clinical evidence for efficacy and safety, which is still sparse. Digital endpoints may be used for clinical trials for DTx. However, validation of digital endpoints and diagnostics are a major challenge in the absence of a gold standard. Currently, there is little experience of using digital endpoints to measure the primary outcome in randomized clinical trials. Also, because of poorly defined exposure (dose), response endpoints, and relationships between exposure and response, the design of clinical trials may not be straightforward. In addition, technical characteristics of the DTx may be rapidly upgraded over the course of the trial, and the technology itself may become outdated before the trial ends. Further, blinding in a digital intervention trial may also be problematic. To add, the use of a placebo control group may be unethical or not feasible in some cases. Furthermore, in a mobile device based clinical research, the remote informed consent process raises privacy concerns. Hit of the "OK" button by a subject cannot be considered sufficient consent.[9] Another challenge related to DTx is in the development of new therapeutic targets. Most introduced DTx products are actually digital transformations or optimizations of existing therapies. Also, we do not exactly understand the mechanism of action of some DTx products for CBT. We have basic questions about how to define new components of DTx related to therapeutic targets. 


\section{Comparison to pharmacotherapy}

DTx will be of great interest to most clinical pharmacologists when used for the treatment of diseases for which pharmacological concepts need to be applied to the development and optimization of treatment regimens. The similarity between pharmacotherapy and DTx is that they are both medical treatment methods. However, the fundamental difference is that the therapeutic effects of DTx are present in the absence of an active substance in the systemic circulation. Therefore, all pharmacokinetic/pharmacodynamic (PK/PD) parameters related to plasma concentrations cannot be derived and used for response optimization. However, similar exposure concepts not based on concentrations can be applied. The dosage, frequency, time (total amount of used time and/or completed percentage out of required sessions), or modules can be all regarded as the exposure of DTx.

The comparisons between pharmacotherapy and DTx from the point of view of clinical pharmacology are summarized in Table 2. In this table, there are several fundamental characteristics of DTx that do not apply to conventional pharmacology.
However, there is an opportunity to apply pharmacology concepts by quantitative modeling (pharmacometrics), because the input and response variables of DTx are all digitally implemented and quantifiable, which was difficult in previous nondigitalized treatments methods.

\section{Exposure-response relationship of digital therapeutics}

Exposure-response relationship is a core principle of clinical pharmacology for developing optimal drug therapy. However, there are few dose-response studies related to DTx. Some of the staff at Pear Therapeutics presented a poster demonstrating dose-dependent outcomes by reSET $^{\oplus}$ at the American Conference on Pharmacometrics 2018, which is the first quantitative analysis of the dose-response relationship related to DTx, although a pharmacodynamics model-based analysis was not explored. The relationship between dose (defined as engagement/module completion) and abstinence (\% of all urine drug screens negative), or appointment attendance ( $\%$ of study visits attended) was analyzed using linear regression. Abstinence was

Table 2. Conceptual comparison between pharmacotherapy and digital therapeutics from the point of view of clinical pharmacology

\begin{tabular}{|c|c|c|}
\hline & Pharmacotherapy & Digital Therapeutics \\
\hline \multicolumn{3}{|l|}{ Pharmacokinetics } \\
\hline Route of administration & Oral, intravenous, or others to systemic circulation & User interface, Input panel \\
\hline Exposure & Dosage, dosing interval & Using time, frequency, duration \\
\hline Systemic exposure measures & AUC, Cmax & Hard to be parameterized \\
\hline Elimination & $\begin{array}{l}\text { Metabolism and Excretion. } \\
\text { Described by half-life or clearance parameter. }\end{array}$ & Hard to be quantified \\
\hline \multicolumn{3}{|l|}{ Pharmacodynamics } \\
\hline Mechanism of action & Clear & Unclear \\
\hline Hysteresis or Tachyphylaxis & Can occur & Can occur \\
\hline Characterizing potency and efficacy & Parameterized as Emax and EC50 & Hard to be parameterized \\
\hline Exposure-Response Relationship & Can be represented with a curve. & Hard to be represented \\
\hline Treatment Regimen & Based on PK/PD, Exposure-Response relationship & Empirical \\
\hline \multicolumn{3}{|l|}{ Safety } \\
\hline Adverse Effect & Toxicity related to target or off-target of the drug & $\begin{array}{l}\text { Related to using mobile device } \\
\text { (Eye, neck, finger, back pain) } \\
\text { Other CNS effects (disturbance on sleep, etc) }\end{array}$ \\
\hline Serious Adverse Events & Can occur & Less likely \\
\hline Hypersensitivity & Can occur & None \\
\hline Addiction & Some drugs & Dependency may occur \\
\hline \multicolumn{3}{|l|}{ Development and Clinical Use } \\
\hline Therapeutic area & Not restricted & Mostly on neuropsychiatric disease \\
\hline Drug Interactions & $\begin{array}{l}\text { PK/PD mechanism based interaction } \\
\text { Unknown for Drug-DTx interactions }\end{array}$ & Unknown \\
\hline Development Strategy & $\begin{array}{l}\text { Unmet medical needs } \\
\text { Target identify }\end{array}$ & $\begin{array}{l}\text { Amplification of existing non-pharmacological } \\
\text { therapy }\end{array}$ \\
\hline Deliver to Patients & $\begin{array}{l}\text { Pharmacy } \\
\text { Pharmacist }\end{array}$ & $\begin{array}{l}\text { App store } \\
\text { No specialist }\end{array}$ \\
\hline
\end{tabular}


moderate $\left(\mathrm{R}^{2}=0.21, \mathrm{p}<.001\right)$, and appointment attendance highly correlated $\left(\mathrm{R}^{2}=0.63, \mathrm{p}<.001\right)$ with dose. Individuals who completed $\geq 24$ modules in the first 6 weeks were over 3 times more likely to complete the entire 12 weeks than patients who completed $<18$ modules (Odds Ratio $=3.28, \mathrm{p}=.003$ ). They concluded that the dose of reSET ${ }^{\circledast}$ correlates with improved treatment outcomes and early engagement was an indicator of treatment retention. They suggested that reSET ${ }^{\circledast}$ may be an outcome predictive tool for enabling better clinical management of SUD patients.[10]

In the near future, the use of pharmacometrics for the exploration of dose-response relationships, and real-world big data analysis will spread widely because DTx can generate large data that can be used for pharmacometrics analysis.

\section{Discussion}

DTx presents both challenges and opportunities for clinical pharmacology. The core principles of clinical pharmacology can be applied to a DTx as long as it has a dose-response relationship. The investigation of dose-response relationships and their application to the designing of optimum therapies is a desirable future direction for DTx. Various types of dose-response relationships are possible for DTx and include Linear or Emax, synergism or antagonism, time-dependent response hysteresis or proteresis, tolerance, feedback regulation, and inverted $U$ shape response (hormesis), characterized by a low dose stimulation and a high dose inhibitory effect. For example, a DTx-based product for CBT is likely to have a hormetic response because the cognitive and/or physical function of the body have/has a limited capacity for therapeutic intervention acceptance.[11] Finding the dose required for optimum exposure (in between low and high doses) to a DTx product during a single session and the total treatment duration may be key factors for the success of clinical trials. Validation of the digital endpoint should be premised before the dose-response of a DTx is analyzed. Inter-individual variability in response to DTx products is another concern. Depending on the phenotypes for the DTx products, responders may be distinguishable from non-responders. Investigation of the factors affecting the response to or outcome of DTx can be the basis for personalized therapy. Potential biomarkers may be identified during this investigation.

An innovative regulatory approval system is needed for the growing number of DTx products, which should be better than the previous application and review system. The Digital Health Software Precertification (Pre-Cert) program has been launched to expedite regulatory review for companies that demonstrate quality and organizational excellence in software development. [12] However, when DTx products are combined with a new chemical or biological entity, drug regulations come first regardless of their intended use. Clinical pharmacology should play an essential role in the conceptual design of these products. The economics of DTx development is beyond the scope of this article, but it is a key driver of development. Also, insurance coverage will be an important factor in clinical use.

Drug-drug interactions and usage for special populations are important subjects in clinical pharmacology review of medicinal products. Interaction effects of DTx (as both perpetrator and victim) will be of concern, as DTx products are being increasingly available amongst mainstream therapeutics. It is possible for DTx products to have significant interactions with medical, surgical, and non-pharmacological treatments, other DTx products, and any other digital technologies such as virtual reality and games. Designing and conducting a clinical study to investigate these interactions of DTx products will be a great challenge to developers and regulators.

DTx is generally considered safe. However, we lack knowledge of the long-term safety of DTx, and serious adverse events can occur when they are combined with drugs or invasive devices. Although there has been no systematic review of the safety of DTx, we can imagine that several harmful effects may be related to the use of these digital devices. The use of mobile devices is likely to induce a strained posture that can lead to musculoskeletal discomfort, and concentration on a display device may lead to audio-visual and central nervous system (CNS) adverse effects. Attention should be paid to real-life accidents that can occur because of the ubiquitous feature of DTx, especially when driving. These concerns warrant further clinical investigations and surveillance of the safety of DTx.

In conclusion, DTx includes software-based therapeutic interventions delivered by a digital interface and has the potential to affect the healthcare landscape. The advancement of DTx will create many opportunities for healthcare professionals, and lead to innovative convergence of medicines, devices, and software. All DTx products must be optimally exposed to ensure efficacy and safety when used as therapeutically indicated. Clinical pharmacology has been the core discipline for developing the right dosing schedule that maximizes therapeutic benefit (by combining quantitative analysis of the right drug, with the right patient, and the right dose). Therefore, clinical pharmacology has great potential in the development, application, and regulation of DTx.

\section{Acknowledgments}

None.

\section{Conflict of interest}

- Authors: The author has no conflict of interest to declare.

- Reviewers: Nothing to declare

- Editors: Nothing to declare

\section{References}

1. FDA permits marketing of mobile medical application for substance use disorder https://www.fda.gov/NewsEvents/Newsroom/PressAnnouncements/ucm576087. htm Accessed 12 February 2019

2. Digital Therapeutics Alliance https://www.dtxalliance.org/ Accessed 10 January 2019 
3. Digital therapeutics https://en.wikipedia.org/wiki/Digital_therapeutics Accessed 10 January 2019

4. Digital Therapeutics: Combining Technology and Evidence-based Medicine to Transform Personalized Patient Care https://www.dtxalliance.org/ wp-content/uploads/2018/09/DTA-Report_DTx-Industry-Foundations.pdf Accessed 10 January 2019

5. Plowman RS, Peters-Strickland T, Savage GM. Digital medicines: clinical review on the safety of tablets with sensors. Expert Opin Drug Saf 2018;17:849-852. doi: 10.1080/14740338.2018.1508447.

6. Voelker R. Digital Pill Gains Approval. JAMA 2018;319:14. doi: 10.1001/ jama.2017.19309.

7. Examples of Mobile Apps For Which the FDA Will Exercise Enforcement Discretion https://www.fda.gov/MedicalDevices/DigitalHealth/MobileMedicalApplications/ucm368744.htm Accessed 12 February 2019

8. Pear Therapeutics receives Expedited Access Pathway designation from FDA for reSET-O ${ }^{\mathrm{TM}}$ prescription digital therapeutic to treat opioid use disorder. <http://www.businesswire.com/news/home/20171018006174/ en/Pear-Therapeutics-Receives-ExpeditedAccess-Pathway-Designation> Accessed 10 January 2019

9. Sverdlov O, van Dam J, Hannesdottir K, Thornton-Wells T. Digital Therapeutics: An Integral Component of Digital Innovation in Drug Development. Clin Pharmacol Ther 2018;104:72-80. doi: 10.1002/cpt.1036.

10. Luderer HF, Coolidge K, Campbell AN, Nunes EV, Maricich YA. reSET Digital Therapeutic for SUD Demonstrates Dose-Dependent Improvement in Outcomes. J Pharmacokinet Pharmacodyn 2018;45:S84-S85.

11. Mattson MP, Calabrese EJ. Hormesis: what it is and why it matters. In: Hormesis. Springer, 2010;1-13.

12. Lee TT, Kesselheim AS. U.S. Food and Drug Administration Precertification Pilot Program for Digital Health Software: Weighing the Benefits and Risks. Ann Intern Med 2018;168:730-732. doi: 10.7326/M17-2715.1 